\title{
Service implications of implementing guidance for oral bowel cleansing agents in colonoscopy
}

\author{
${ }^{1}$ PK Flanagan, ${ }^{2} \mathrm{MS}$ Ahmed, ${ }^{3} \mathrm{SM}$ Tin, ${ }^{4} \mathrm{~S}$ Sarkar \\ ${ }^{1}$ Gastroenterology Specialist Registrar, Department of Gastroenterology; ${ }^{2}$ Consultant Nephrologist, Department of Renal Medicine and \\ Transplantation; ${ }^{3}$ Senior Pharmacist, Pharmacy Department, ${ }^{4}$ Consultant Gastroenterologist and Endoscopist, Department of Gastroenterology, \\ Royal Liverpool University Hospital, Liverpool, UK
}

\section{ABSTRACT}

Introduction:Bowel preparation has led to serious adverse events. Consequently, regulatory and advisory bodies have issued guidance, including a risk stratification of patients, aiming to minimise this risk.

Aims: To determine the impact of the new guidance on our current practice of Picolax used in colonoscopy with regard to patient stratification, compliance and service implementation.

Methods: Patients listed for day case colonoscopy during October 2009 were audited using data from the hospital and endoscopy databases. A follow-up, structured patient telephone interview evaluated side effects, the assessment process and patient experience.

Results: Of the participating I 12 patients, $97.4 \%$ were clinically reviewed before colonoscopy, $98.3 \%$ received written instructions and $70 \%$ verbal instructions. None had their preparation dispensed by a regulated professional. Although $62 \%$ of patients were retrospectively identified as 'at risk' (elderly, with congestive cardiac failure, cirrhosis or chronic kidney disease or on angiotensin-converting enzyme inhibitors, angiotensin receptor blockers, diuretics or non-steroidal anti-inflammatory drugs), none were identified before their procedure. Significant events (postural hypotension, collapse, dizziness, palpitations, faint) were seen in $4.4 \%$, all of whom could potentially be identified as 'at risk' using a stratification of low estimated glomerular filtration rate* with relevant co-morbidity (congestive cardiac failure or cirrhosis). Conclusions: Given the difficulties with compliance and the high prevalence of 'at risk' patients, the implementation will be challenging and require significant service redesign.

KEYWORDS Adverse events, bowel preparation, colonoscopy, complications, oral bowel cleansing agents, safety
Correspondence to S Sarkar, Department of Gastroenterology, Royal Liverpool University Hospital, Prescot Street, Liverpool L9 8XL, UK

tel. +44 (0) I5I 7063554 e-mail sanchoy@aol.com

DECLARATION OF INTERESTS No conflict of interests declared.

\section{INTRODUCTION}

The ability to perform high-quality colonoscopy depends on a number of variables, including good quality bowel preparation.' Several different oral bowel cleansing agents are widely used for this purpose, which can be broadly divided into non-absorbed isosmotic agents (i.e. polyethylene glycol [PEG]-based preparations), hyperosmolar agents (sodium phosphate, magnesium compounds) and stimulants (sodium picosulpate, senna). Sodium picosulphate is by far the most commonly used hyperosmolar agent in the UK, probably on the basis of cost and tolerability compared with the more expensive, larger-volume PEG-based preparations.

The National Patient Safety Agency (NPSA) issued an alert in 2009 that highlighted 218 incidents of harm arising directly from the use of oral bowel cleansing agents and concerns over their prescription and distribution. ${ }^{2}$ Consequently, a number of central directives issued to National Health Service trusts stipulated that all patients should be reviewed by a healthcare professional (medical doctor or nurse) before the issue of an oral bowel cleansing agent; the agent should be prescribed by a healthcare professional (medical doctor or registered nurse with prescribing certification); that patients should be given verbal and written information on its safe use; and that it should be dispensed by an authorised professional (medical doctor, registered nurse or pharmacist).

There is a relative paucity of trial data on the safety of oral bowel cleansing agents. A recent systematic review of 82 clinical trials did not find any significant adverse events, although serum electrolyte imbalances were noted. ${ }^{3}$ Other studies have not only highlighted the latter, but also a deterioration in renal function and even renal injury with these agents. ${ }^{4}$ However, a large metaanalysis did not find evidence of a significant change in renal function in otherwise healthy patients, and other studies have suggested that renal function deterioration is limited to those with pre-existing risk factors for renal dysfunction or elderly patients. ${ }^{5-7}$

There is, however, evidence of significant electrolyte imbalance, particularly from sodium phosphate-based 
oral preparation, in patients with normal creatinine; the incidence for this is increased in the elderly. ${ }^{8-10}$ In addition, acute phosphate nephropathy and acute and chronic renal failure have been described." The concomitant use of other medications such as nonsteroidal anti-inflammatory drugs (NSAIDs), diuretics, angiotensin-converting enzyme (ACE) inhibitors and angiotensin receptor blockers (ARBs) may increase the risk. ${ }^{7,812}$ Patient co-morbidity, particularly congestive cardiac failure (CCF), advanced liver disease and renal failure, has been suggested to be an additional independent risk factor. ${ }^{13,14}$ However, there have been no reports of renal dysfunction or electrolyte imbalance with PEGbased oral bowel cleansing agents. ${ }^{13}$

The recently published Consensus guidelines for the prescription and administration of oral bowel cleansing agents (jointly developed by the Royal College of Surgeons, British Society of Gastroenterology, British Society of Gastrointestinal and Abdominal Radiology, Renal Association and the Royal College of Radiologists) recommends an eight-step process for all patients when prescribing oral bowel cleansing agents. ${ }^{15}$ These include clinical review, measurement of estimated glomerular filtration rate (eGFR), a review of medications and an assessment for co-morbidities such as chronic kidney disease (CKD), CCF, liver cirrhosis or hypertension.

Both the UK and US consensus guidelines have defined 'at risk' patients as those taking diuretics, NSAIDs, ACE inhibitors or ARBs, patients with low eGFR (<60 ml/min) and those with significant co-morbidities (cirrhosis, heart failure and renal dysfunction). ${ }^{13,15}$ Both guidelines suggest the use of PEG-based solutions in patients with one or more of these risk factors.

Compliance with the NPSA guidance and the UK consensus guidelines may result in a significant burden of resource, cost and service redesign for most healthcare trusts in the UK. The aims of this study in patients undergoing colonoscopy were:

- To assess our compliance with the guidance.

- To determine the potential service implications.

- To determine the effect of bowel preparation on our patients, including side effects and adverse events.

- To determine the impact of the 'at risk' stratification for bowel preparation administration in our patient population.

\section{METHODS}

\section{Study design}

This project was registered and approved by the trust audit committee. It was a retrospective snapshot audit with a 30-day follow-up telephone interview of patients who had a colonoscopic procedure performed in a onemonth period at the Royal Liverpool University Hospital.

\section{Data collection}

All patients who were either listed or underwent a colonoscopy between I October and 31 October 2009 were identified from the hospital booking system. All data collection was performed in November 2009. A standard form captured procedural data of the colonoscopy obtained from the endoscopy database, demographic data from the hospital booking system, blood results from hospital lab systems and information attained at telephone interview with each patient. For data protection and patient confidentiality purposes, the only patient-identifying heading was a unique identification number on the database which was stored on a secure hospital network drive. Following data entry all forms which contained patient-identifying information were securely destroyed.

\section{Telephone interview}

Informed verbal consent was obtained prior to the telephone interview. Patients were interviewed in chronological order to reduce recall bias. To avoid selection bias, cancelled or failed-to-attend patients were included. Patients were contacted on one further occasion if there was no initial response. If still unavailable, a contact telephone number was left where possible. Patients were deemed 'non-responders' if they were not contactable by these methods. The telephone interviews were conducted by three gastroenterologists using the questionnaire in Table I. The questions assessed compliance with the NPSA guidance, patient co-morbidity, medications, pre-procedure experience, procedural experience and experience of bowel preparation, including any side effects or adverse events.

\section{Inclusion criteria}

All patients aged above 18 years listed for a colonoscopy with a bowel preparation regime containing sodium picosulphate (two sachets of Picolax and two tablets of Senna [the majority] or three sachets of Picolax [Bowel Cancer Screening Programme patients]) were eligible. Patients who gave verbal consent and completed the telephone interview were included. Patients who received Klean Prep as their bowel preparation were excluded because the extremely small number of patients would inevitably be open to bias. Patients who did not give verbal consent, did not take the bowel preparation or were non-responders were excluded due to incomplete datasets.

\section{Definitions}

Side effects: any unpleasant symptom reported by the patient after Picolax, other than increased bowel frequency or diarrhoea.

Significant event: symptoms considered to be related to cardiovascular disturbance, including hypotension, such as dizziness, palpitations, collapse, fainting, myocardial infarction, stroke and death. 


\section{TABLE I The telephone questionnaire}

\begin{tabular}{|c|c|c|}
\hline Question & Purpose & Scale \\
\hline $\begin{array}{l}\text { Did you attend } \\
\text { your outpatient } \\
\text { department? } \\
\text { If not why not? }\end{array}$ & $\begin{array}{l}\text { Determine } \\
\text { if the patient } \\
\text { did not attend } \\
\text { because of the } \\
\text { bowel prep or } \\
\text { its side effects }\end{array}$ & $\begin{array}{l}\text { Yes/No (free text } \\
\text { response) }\end{array}$ \\
\hline $\begin{array}{l}\text { Did you see a healthcare } \\
\text { professional before the } \\
\text { day of your test? }\end{array}$ & $\begin{array}{l}\text { Check NPSA } \\
\text { compliance }\end{array}$ & $\begin{array}{l}\text { No/Yes (doctor, } \\
\text { nurse, GP) }\end{array}$ \\
\hline $\begin{array}{l}\text { Did you get written } \\
\text { information about taking } \\
\text { your bowel prep? }\end{array}$ & $\begin{array}{l}\text { Check NPSA } \\
\text { compliance }\end{array}$ & Yes/No \\
\hline $\begin{array}{l}\text { Did you get verbal } \\
\text { information on your } \\
\text { bowel prep? }\end{array}$ & $\begin{array}{l}\text { Check NPSA } \\
\text { compliance }\end{array}$ & Yes/No \\
\hline $\begin{array}{l}\text { List your medical } \\
\text { conditions. }\end{array}$ & $\begin{array}{l}\text { Determine 'at } \\
\text { risk' patients }\end{array}$ & $\begin{array}{l}\text { Yes/No response } \\
\text { to a list of medical } \\
\text { conditions }\end{array}$ \\
\hline List your medications. & $\begin{array}{l}\text { Determine 'at } \\
\text { risk' patients }\end{array}$ & $\begin{array}{l}\text { Yes/No response } \\
\text { to a list of } \\
\text { medications }\end{array}$ \\
\hline $\begin{array}{l}\text { How did the bowel } \\
\text { prep impact your life? }\end{array}$ & $\begin{array}{l}\text { Determine } \\
\text { patient } \\
\text { experience }\end{array}$ & $\begin{array}{l}\text { Not at all/Little/ } \\
\text { Moderately/A } \\
\text { lot/Huge effect }\end{array}$ \\
\hline $\begin{array}{l}\text { Would the bowel prep } \\
\text { affect the decision to } \\
\text { have the test again? }\end{array}$ & $\begin{array}{l}\text { Determine } \\
\text { patient } \\
\text { experience }\end{array}$ & $\begin{array}{l}\text { No/Mild effect } \\
\text { (would have } \\
\text { test)/Moderate } \\
\text { - (only if } \\
\text { required)/ } \\
\text { Significant (would } \\
\text { avoid)/Would } \\
\text { never take prep }\end{array}$ \\
\hline $\begin{array}{l}\text { Did you experience } \\
\text { any side effects with } \\
\text { the bowel prep? If so, } \\
\text { what, and whom did } \\
\text { you consult? }\end{array}$ & $\begin{array}{l}\text { Determine } \\
\text { side effects, } \\
\text { adverse events } \\
\text { and severity of } \\
\text { event }\end{array}$ & $\begin{array}{l}\text { Yes (free text } \\
\text { response)/No }\end{array}$ \\
\hline $\begin{array}{l}\text { How would you rate } \\
\text { the discomfort you } \\
\text { experienced during } \\
\text { your procedure? }\end{array}$ & $\begin{array}{l}\text { Determine } \\
\text { patient } \\
\text { experience }\end{array}$ & $\begin{array}{l}\text { None/Minimal/ } \\
\text { Mild/Moderate/ } \\
\text { Severe }\end{array}$ \\
\hline
\end{tabular}

NPSA compliance: compliance with four recommendations made by NPSA (clinical assessment prior to prescription of bowel preparation, authorisation of bowel preparation by clinical staff, an explanation of the bowel preparation to every patient, an authorised professional dispenses the medication with verbal and written explanation).

Consensus guidelines compliance: compliance with the NPSA guidance and, in addition, evaluation of the patient with blood test results (eGFR), medications of ACE inhibitors, ARBs, diuretics, NSAIDs and assessment for 'at risk' conditions prior to colonoscopy.

'At risk' patients: elderly patients (>65 years) or patients with medical conditions or on regular medication on which the NPSA and consensus guidelines advised caution
TABLE 2 Patient demographics

\begin{tabular}{|l|l|}
\hline Patient numbers & $\mathrm{I}$ I2 \\
\hline Male/female ratio & $\mathrm{I} .4: \mathrm{I}$ \\
\hline Mean age & 62.5 years \\
\hline Elderly patients (>65 years) & $56(50 \%)$ \\
\hline Low eGFR (<60 ml/min) & $28(31 \%)(\mathrm{n}=89)$ \\
\hline No eGFR result & $23(20.5 \%)$ \\
\hline 'At risk' medication & $23(20.5 \%)$ \\
\hline 'At risk' co-morbidities & $23(20.5 \%)$ \\
\hline $\begin{array}{l}\text { Number of 'at risk' patients (elderly, low } \\
\text { eGFR, medications, co-morbidity) }\end{array}$ & $69(62 \%)$ \\
\hline Total number of side effects & $27(24 \%)$ \\
\hline Total number of significant events & $5(4.4 \%)$ \\
\hline
\end{tabular}

when administering with oral bowel cleansing agents. This included patients with CKD, CCF, hypertension, cirrhosis and diabetes or taking regular diuretics, ACE inhibitors,ARBs, NSAIDs or drugs that caused the syndrome of inappropriate antidiuretic hormone secretion. eGFR values: 'low eGFR' is defined as $<60 \mathrm{ml} / \mathrm{min}$.

30-day mortality: death for any reason within 30 days of either taking oral bowel preparation or having a colonoscopy.

Emergency readmission: unplanned hospital admission due to the oral bowel preparation or within six days following the colonoscopy.

\section{RESULTS}

\section{Patient participation}

Of the 265 elective colonoscopies scheduled, the following patients were excluded: 79 because their contact details were unobtainable, 69 because they were non-responders, three because they had Klean Prep due to poor preparation with Picolax previously and two as they did not take any bowel preparation and did not attend for colonoscopy. This left 112 patients with a complete dataset ( $42 \%$ sample size). Of those who participated, the mean age was 62.5 years (standard deviation [SD] 14.5) and 58\% were male (Table 2).

\section{Procedural data and patient outcome}

Colonoscopy (98.2\% day case, $89.3 \%$ diagnostic) was successfully completed in 98 patients (87.5\%). The 14 failures were due to technical reasons in seven (e.g. looping), the pathology encountered in four, and poor bowel preparation in three. In total, 81 colonoscopies (72.3\%) were performed by consultants, six $(5.4 \%)$ by nurse endoscopists, 13 (1 I.6\%) by independent trainees and $12(10.7 \%)$ by supervised trainees.

Sedation was used in $10 \mathrm{I}$ patients $(90.2 \%)$. Midazolam was used in 100 patients at a mean sedative doses of $2.05 \mathrm{mg}$ ( $\mathrm{l} .7 \mathrm{I} \mathrm{mg}$ in patients $\geq 70$ years). Pethidine was used in 57 patients (5I\%) at mean doses of $39.6 \mathrm{mg}$ (3I.8 $\mathrm{mg}$ for 


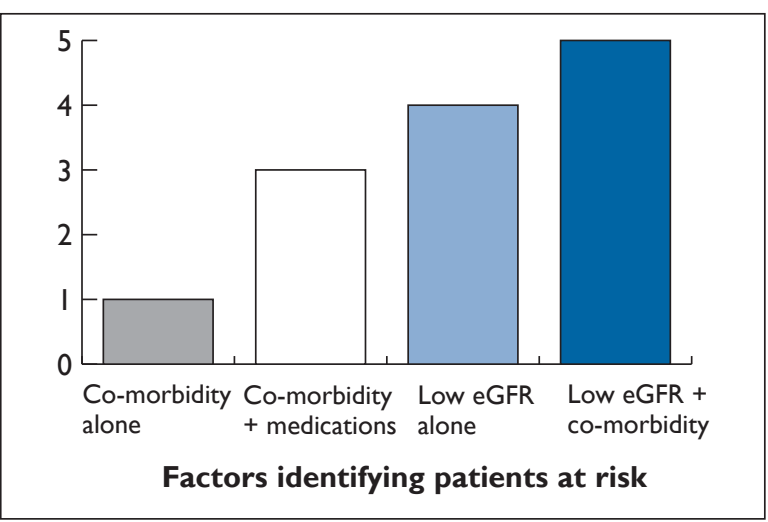

FIGURE I The impact of risk stratification on predicting side effects and significant events in patients who had taken sodium picosulphate.

those $\geq 70$ years). Fentanyl was used in 43 patients (38.4\%) at a mean dose of $72.7 \mathrm{mg}$ (58.3 $\mathrm{mg} \geq 70$ years).

The endoscopist rated the bowel preparation as 'poor' in 12 patients ( $11 \%$ ), thus making the procedure difficult to interpret. There were no emergency readmissions or 30-day mortality.

\section{Patient experience of bowel preparation}

The overall impact on lives scored a median response of three (moderate impact). In terms of expectation, $64 \%$ reported 'as expected', $22.8 \%$ 'better' or 'much better than expected' and 13.2\% 'worse' or 'much worse'. When asked whether the need for bowel preparation would affect their decision to have a repeat colonoscopy, $59.1 \%$ reported 'no effect', $35.7 \%$ 'would take it if needed' and $5.2 \%$ would 'avoid it'.

\section{NPSA compliance}

Clinical evaluation before colonoscopy was achieved in $97.4 \%$ of patients. Patients received written guidance on bowel preparation in $98.2 \%$ of cases and verbal instruction in $71.3 \%$. In all cases, the correct prescription authorisation was achieved, but none had their bowel preparation dispensed by authorised professional personnel.

\section{Consensus guidelines compliance}

No patients had their blood tests reviewed or were identified as 'at risk' prior to their procedure with regard to their eGFR, medications or co-morbidities.

\section{Retrospective stratification for at risk patients}

Retrospective stratification revealed that $20.5 \%$ of patients had 'at risk' co-morbidities and $20.5 \%$ were taking 'at risk' medications. Fifty per cent were elderly. There was no traceable eGFR value in $20.5 \%$ and in the remainer $31.4 \%$ had a low eGFR. Overall, the total number of patients that can be classified retrospectively as 'at risk' was 69 (62\%).
Side effects, significant events and predicting factors Overall, 27 patients (23.4\%) reported side effects and, although the median severity response was 'none', II\% reported either 'moderate' or 'severe'. The five patients (4.4\%) who had significant events all received two sachets of Picolax and were in the 'at risk' group. These five patients were further analysed to show the relative contribution of the various risk factors. Using comorbidity alone would have defined one patient as 'at risk', combining co-morbidity with medications would have defined three, using low eGFR alone would have defined four, but combining low eGFR with 'at risk' co-morbidity would have been defined all five patients as at risk (Figure I).

\section{DISCUSSION}

Our study has highlighted a number of challenges faced by the majority of endoscopy units in the UK regarding compliance with the NPSA and consensus guidelines for the administration of oral bowel cleansing agents in lower gastrointestinal endoscopy.

This study has also highlighted that, from a patient experience and outcome perspective, our oral bowel cleansing agent results with sodium picosulphate (Picolax) were possibly substandard, given that 13\% of our patients reported their experience of taking the preparation as 'worse' or 'much worse than expected' and $5.2 \%$ said they would avoid future colonoscopy because of the preparation. Furthermore, in $11.5 \%$ of colonoscopies the bowel preparation was poor, suggesting that the interpretation of the results in these patients should be done cautiously due to issues of missed pathology and so on. Poor bowel preparation accounted for $14 \%$ of the colonoscopy failures.

In order to achieve NPSA compliance, we instigated a number of initiatives, which helped to attain high compliance in three of its four directives. These included an electronic referral system with referrer confirmation of patient fitness and a prescription of the bowel preparation. Unfortunately, we could not comply with the directive of authorised professionals dispensing the bowel preparation as this is done by our administrative and clerical (A\&C) staff, which we suspect is similar to the majority of endoscopy units and radiology departments in the UK. With about 7,000 lower gastrointestinal endoscopic procedures per year, it would not be practical to comply with this recommendation. We therefore adopted a riskminimising strategy developed in conjunction with our pharmacy department, whereby A\&C staff work to an individualised patient dispensing checklist before the bowel preparation is issued.

Our study has highlighted that there would have to be significant redesign of our service to comply with the consensus guidelines as none of our patients had their 
blood results, medications or co-morbidities assessed prior to their procedure. The retrospective review of our patient population showed that while more than $20 \%$ had no recorded eGFR, $64 \%$ could still be considered 'at risk' and would support the guidelines that all patients should be assessed systematically.

The other main finding of our study was that $23 \%$ of patients experienced some sort of side effect to the bowel preparation and, of these, five patients (4.4\%) had significant events, even in this snapshot audit. The significant events all occurred in 'at risk' patients. Retrospective analysis of these patients suggests that some risk factors may be of greater value than others in predicting patients who may suffer adverse events (Figure I).

While we can acknowledge a number of limitations in this audit - the small number of events and patients audited leading to bias from type II errors, the definition of significant events, a sample size of less than $50 \%$, its retrospective nature, the bias of recall and selection of patients, co-founding factors such as the effect of sedation on patient recall, the lack of poor outcomes and so on - our audit at least has presented data reported by unselected patients both with and without side effects from the bowel preparation.

Given the acknowledged limitations of this study, it is not possible to make a firm conclusion about the validity of the risk stratification algorithm. However, this study suggests that adopting a strategy of defining 'at risk' patients and attaining eGFR in patients prior to listing for lower gastrointestinal endoscopy may help to identify patients who are more likely to experience adverse events with sodium picosulphate oral bowel cleansing.

It also suggests that risk stratification may be achieved by combining eGFR with a patient-completed list of co-morbidities without the need for a medication review or adding age to the algorithm. In addition, patients' medication details are often scant and making presumptions regarding their prescriptions for the purpose of identifying 'at risk' patients is best avoided. This approach would require an appropriately powered prospective study to validate its findings. However, with the recent publication of the consensus guidelines, the relevance of performing such a study can be debated.

Patients who require either a regular screening or surveillance colonoscopy (outside the Bowel Cancer Screening Programme) may be particularly challenging for compliance with the NPSA and consensus guidelines.,15 This is because these patients are automatically scheduled from protocols based on their last colonoscopy and therefore do not have a recent clinic consultation (usually there are no recent assessments for the appropriateness of the test and the patient's fitness to undergo the bowel

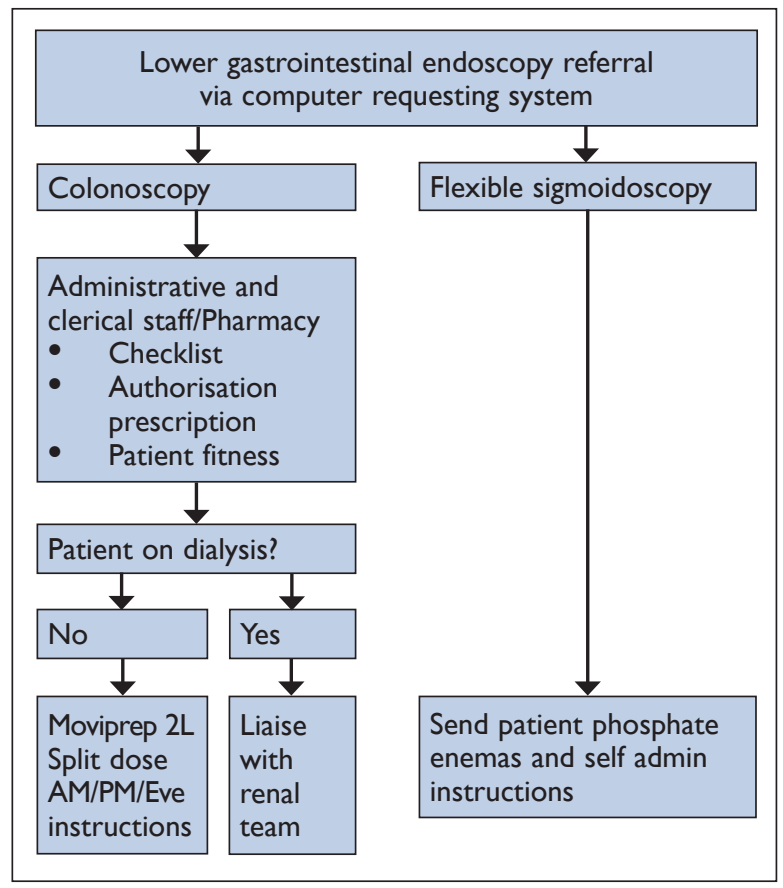

FIGURE 2 Our new protocol for bowel preparation administration in lower gastrointestinal endoscopy.

preparation and no determination of risk factors or of whether the patient has a recently authorised prescription). One potential method of overcoming these issues would be a nurse-led assessment/protocol clinic for these patients, which would vastly improve both compliance and, potentially, patient safety.

For the vast majority of our patients, however, compliance will not be met even with these mechanisms, as future development of nurse-led pre-assessment clinics for all patients undergoing lower gastrointestinal endoscopy is unlikely to be funded, given the number of procedures and the costs involved in setting up and maintaining such a service.

A risk stratification approach without a pre-assessment clinic would not only mean a significant service redesign with resource implications, but also overwhelming practical difficulties including funding additional blood tests (eGFR), developing mechanisms for identifying 'at risk' patients, developing protocols to follow up patients' blood test results and interpreting them, defining responsibilities for the patient pathway such as decisions on authorising alteration in bowel preparation regimes and so on.

We therefore developed an alternative strategy in collaboration with our pharmacy and renal departments on the basis that a previous audit highlighted inadequate bowel preparation at colonoscopy with our current regime of Senna plus two sachets of Picolax (in this audit greater than 10\%) and that, according to the UK consensus and US consensus guidelines, ${ }^{13,15}$ if a patient is 
deemed 'at risk' the only change to management is the recommendation to use a PEG-based oral bowel cleansing agent. Given that PEG-based preparations can be used even in 'at risk' patients, we postulate that measuring eGFR is clinically unnecessary, unpractical and potentially costly if we move universally to a PEG-based cleansing agent.

We developed a protocol for bowel preparation in lower gastrointestinal endoscopy that would:

- improve the quality of bowel preparation;

- help comply with guidance;

- improve patient safety;

- be practically achievable; and

- be financially viable.

Our cost analysis* showed that switching to a PEG-based preparation was equivalent and cheaper than the unlicensed combination of three sachets of Picolax with eGFR measurement. Consequently, on the recommendation of our renal department, we adopted a split-dose Moviprep regime with $50 \%$ less volume than Klean Prep. This could be administered to the majority of patients with CKD, with special consideration for those on dialysis. Some of the increased cost would be offset by switching from Picolax to phosphate enema preparation for flexible sigmoidoscopy (Figure 2).

\section{CONCLUSION}

Units in the UK that use sodium picosulphate or oral sodium phosphate-based bowel preparations face a number of challenges with significant service redesign required to comply with safety guidance for oral bowel cleansing agents, especially given the high prevalence of at risk patients. Potentially, risk stratification using patient-reported co-morbidities and eGFR may predict adverse events with non-PEG-based cleansing agents, although further evaluation for this is required. Our solution of a low-volume PEG agent without eGFR measurement may offer a more practical alternative strategy to overcome some of the issues.

Acknowledgement The authors would like to thank Dr Sajjid Mahmood and Dr Muhammed Naseer for their help with the data collection.

\section{REFERENCES}

I Froehlich F, Wietlisbach V, Gonvers J] et al. Impact of colonic cleansing on quality and diagnostic yield of colonoscopy: the European Panel of Appropriateness of Gastrointestinal Endoscopy European multicenter study. Gastrointest Endosc 2005; 61:378-84. doi:10.1016/S0016-5107(04)02776-2

2 National Patient Safety Agency. Reducing risk of harm from oral bowel cleansing solutions. London: NPSA; 2009. Available from: http://www.nrls.npsa.nhs.uk/alerts/?entryid45=59869

3 Belsey J, Epstein O, Heresbach D. Systematic review: oral bowel preparation for colonoscopy. Alimen Pharmacol Ther 2007; 25:37384. doi: I0.1 III/j. I365-2036.2006.032I2.x

4 Ahmed M, Raval P, Buganza G. Oral sodium phosphate catharsis and acute renal failure. Am J Gastroenterol 1996; 91:I26I-2.

5 Brunelli SM. Association between oral sodium phosphate bowel preparations and kidney injury: a systematic review and metaanalysis. Am J Kidney Dis 2009; 53:448-56. doi:10.1053/j. ajkd.2008.09.022

6 Seol DC, Hong SN, Kim JH et al. Change in renal function after sodium phosphate preparation for screening colonoscopy. World J Gastroenterol 2010; 16:2010-6. doi:10.3748/wjg.v16.il6.2010

7 Khurana A, McLean L, Atkinson S et al. The effect of oral sodium phosphate drug products on renal function in adults undergoing bowel endoscopy. Arch Intern Med 2008; 168:593-7. doi:10.1001/ archinte. 168.6 .593

8 Ainley EJ, Winwood PJ, Begley JP. Measurement of serum electrolytes and phosphate after sodium phosphate colonoscopy bowel preparation: an evaluation. Digest Dis Sci 2005; 50:1319-23. doi: 10.1007/s 10620-005-2780-9

9 Lieberman DA. Ghormley J, Flora K. Effect of oral sodium phosphate colon preparation on serum electrolytes in patients with normal serum creatinine. Gastrointest Endosc 1996; 43:467-9. doi:10.1016/S0016-5107(96)70287-0

10 Beloosesky Y, Grinblat J, Weiss A et al. Electrolyte disorders following oral sodium phosphate administration for bowel

cleansing in elderly patients. Arch Intern Med 2003; 163:803-8. doi: 10.100I/archinte.163.7.803

II Markowitz GS, Stokes MB, Radhakrishnan J et al.Acute phosphate nephropathy following oral sodium phosphate bowel purgative: an underrecognized cause of chronic renal failure. J Am Soc Nephrol 2005; 16:3389-96. doi:I0.168I/ASN.2005050496

12 Singal AK, Rosman AS, Post JB et al. The renal safety of bowel preparations for colonoscopy: a comparative study of oral sodium phosphate solution and polyethylene glycol. Alimen Pharamcol Ther 2008; 27:4 I-7. doi:10. I I I I/j. I365-2036.2007.03558.x

I3 Wexner SD, Beck DE, Baron TH et al. A consensus document on bowel preparation before colonoscopy: prepared by a task force from the American Society of Colon and Rectal Surgeons (ASCRS), the American Society for Gastrointestinal Endoscopy (ASGE), and the Society of American Gastrointestinal and Endoscopic Surgeons (SAGES). Gastointest Endosc 2006; 63:894909. doi:10.1016/j.gie.2006.03.918

I4 Sica DA, Carl D, Zfass AM. Acute phosphate nephropathy: an emerging issue. Am J Gastroenterol 2007; 102: 1844-7. doi: I0. I I I I/ j.1572-0241.2007.01047.x

15 Connor A, Tolan D, Hughes S et al. Consensus guidelines for the prescription and administration of oral bowel cleansing agents. London: Royal College of Radiologists; 2009. Available from: http://www. library.nhs.uk/GuidelinesFinder/ViewResource.aspx?resID=328025

\section{NOTE}

* The local costings of eGFR were $€ 3.65$, two sachets of Picolax $£ 3.50$ and three sachets of Picolax $£ 7.29$. Moviprep was $£ 10.57$, Klean Prep $€ 10.5 \mathrm{I}$ and phosphate enema $£ 0.50$. The cost per 1,000 colonoscopies for four bowel preparation regimes were $£ 8,390$ for eGFR plus two sachets of Picolax with Senna, $£ 10,940$ eGFR plus three sachets of Picolax, $£ 10,570$ for Moviprep and no eGFR and $£ 10,510$ for Klean Prep and no eGFR. 